\title{
Membrane Spin Up in a Normal Gravity Field: Experiments and Simulations
}

\author{
M. Delapierre* and S. Pellegrino ${ }^{\dagger}$ \\ California Institute of Technology, Pasadena, CA 91125
}

\begin{abstract}
Finite element simulations and experimental observations of the spin up in vacuum of a thin membrane loaded by gravity are presented. The numerical techniques take into account the run time of each simulation and energy convergence; it is shown that accurate results can be obtained quite quickly in a rotating reference frame, and that including stiffness-proportional material damping helps convergence of the integration. It is also found that a very fine finite element mesh around the hub of the membrane is required to obtain consistent results. The experimental setup allows spinning of the membrane in a vacuum box; a measurement technique that uses stereo Digital Image Correlation is presented. A comparison between experiments and simulations using characteristic parameters of the shape of a membrane, i.e. the number of rotational symmetric waves, the average deflection, and the elastic bending strain energy of the membrane, shows good agreement between experiments and simulations.
\end{abstract}

\section{Introduction and Background}

Thin membranes provide larger area, light structures that can be packaged tightly for launch, for applications that include space-based antennas and solar sails. A key requirement is being able to apply a state of prestress to stabilize the deployed shape of the structure. Frequently, prestressing is done by applying corner forces through a set of deployable booms, but there is an increasing interest in lighter and potentially simpler solutions, through the use of centrifugal force. This approach was recently demonstrated by the Ikaros solar sail. ${ }^{1}$

Our long term interest is to characterize the dynamic behavior of large membrane structures spinning in space, through numerical simulations and laboratory experiments. Previous work in this field is rather limited. The natural frequencies of vibration of thin spinning plates (floppy disks) were studied by Bogy and co-workers, ${ }^{2}$ membranes with negligible bending stiffness were analyzed by Eversman ${ }^{4}$ and Simmonds. ${ }^{5}$ To our knowledge, the first experimental study of spinning membranes with very low bending stiffness was carried out by Okuizumi. ${ }^{6}$ In this study, the natural frequencies of axisymmetric modes were found experimentally using a single point laser vibrometer and the structure was excited by shaking the central hub. We too are interested in these effects, but before attempting this kind of experiments, we have carried out a detailed study of the process of membrane spin up, which is significantly affected by the presence of gravity. This is the topic of the present paper.

Consider a circular membrane, attached to a small, central rigid hub. Under static conditions, due to gravity the membrane takes the shape of a taco shell. To avoid high strains that may plastically deform the membrane, we put a horizontal support plate under the membrane, a few centimeters below the hub. This support plate will also have the function of uniformly illuminating the membrane as we are going to describe in Section III.A. Then the membrane hub is rotated about a vertical axis, with a linearly increasing angular velocity, when the required angular velocity is reached, it is kept constant. It will be shown in this paper that the dynamic behavior of the membrane is characterized by a series of successive shape instabilities, as the membrane surface is deformed into a wavy shape with an increasing number of rotationally symmetric waves. Eventually, an axisymmetric shape is reached. These instabilities were also noticed by Okuizumi, ${ }^{7}$

\footnotetext{
*Graduate student, Graduate Aerospace Laboratories, 1200 E. California Blvd, MC 105-50. delapmel@caltech.edu

$\dagger$ Joyce and Kent Kresa Professor of Aeronautics and Professor of Civil Engineering, Graduate Aerospace Laboratories, 1200 E. California Blvd, MC 301-46. AIAA Fellow. sergiop@caltech.edu
} 
who provided a criterion for the first instability of a flat spinning structure. In this paper we quantify these effects, and particularly the angular velocities at which these instabilities occur during spin up and measure the successive shapes experimentally.

The particular dimensions listed in table 1 were chosen, from a dimensional analysis presented in a previous publication. ${ }^{3}$ These dimensions where chosen to achieve a structure dominated by centrifugal force effects and with negligible gravity effect on the natural frequencies and modes shapes (less than $10 \%$ difference among the vibration frequencies with and without gravity).

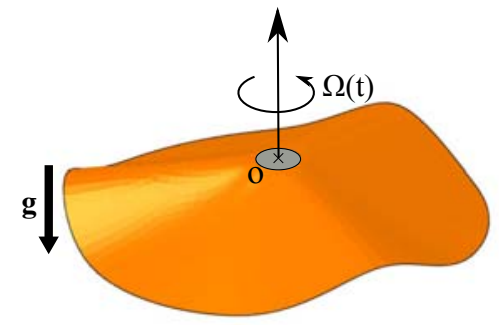

Figure 1: Spin up in a normal gravity field.

\begin{tabular}{|l|c|}
\hline Young's modulus $E$ & $2.5 \mathrm{GPa}$ \\
\hline Poisson's ratio $\nu$ & 0.34 \\
\hline Density $\rho$ & $1420 \mathrm{~kg} \mathrm{~m}^{-3}$ \\
\hline Thickness $h$ & $50 \mu \mathrm{m}$ \\
\hline Outer radius $b$ & $20 \mathrm{~cm}$ \\
\hline Inner Radius $a$ & $2 \mathrm{~cm}$ \\
\hline
\end{tabular}

Table 1: Membrane geometry and material properties.

This paper consists of three parts. In the first part we present the numerical techniques used to accurately simulate the spin up, taking into account the run time of each simulation and energy convergence past each instability. First we find that simulations in a rotating reference frame are orders of magnitudes faster than simulations in a stationary reference frame. Then we show that, although using an energy conservative integration scheme would improve the energy convergence across each instability, the computations take far too long for this approach to be applied. Instead, we include stiffness proportional material damping to help the integration and represent reality more accurately. We also find that using a very fine finite element mesh around the hub (where the stress is concentrated) is necessary to obtain consistent results. In the second part we present the experimental setup and the measurement technique, using stereo Digital Image Correlation. To mitigate air effects we use a low vacuum chamber. With this setup we were able to measure similar shape instabilities to those predicted by the simulations. We also present solutions to some of the challenges associated with using stereo DIC for measuring the shapes of spinning, transparent and very flexible structures. The last part of the paper presents a comparison between experiments and simulations using characteristic parameters of the shape of a membrane, i.e. the number of rotational symmetric waves, the average deflection, and the elastic bending strain energy of the membrane. We find good agreement between experiments and simulations.

\section{Finite Element Simulations}

Results of nonlinear dynamic implicit integrations performed with Abaqus/Standard finite element software, to simulate the large deflection and the instabilities of the membrane during spin up are presented. First we compare simulations in a stationary reference frame to simulations in a membrane-mounted (spinning) reference frame. Then stress concentrations around the hub are investigated by considering a finer finite element mesh. Lastly energy convergence across the instabilities and the time required to run simulations of different accuracy levels is considered. 
We consider a linear elastic material with the properties of Kapton film and in some simulations we introduce stiffness proportional damping. All simulations are carried out with S4R elements: 4-node doubly curved thin shell elements with reduced integration, hourglass control, finite membrane strains, 5 thickness integration points elements. They are numerical efficient elements based on the Kirchhoff shell theory. We simulate the central hub by rigidly constraining the nodes along the inner radius circle. Lastly, we test the integration scheme by running simulations without the support plate, with the acceleration profile in figure 2 , and later we include a frictionless support plate $3.4 \mathrm{~cm}$ below the hub and use the experimental acceleration profile shown in figure 3 .

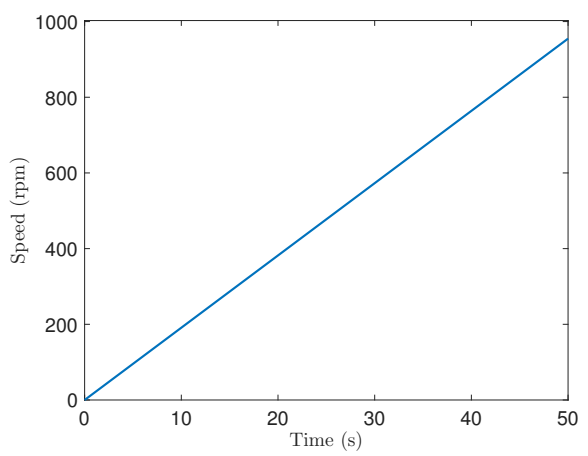

Figure 2: Spin up profile 1.

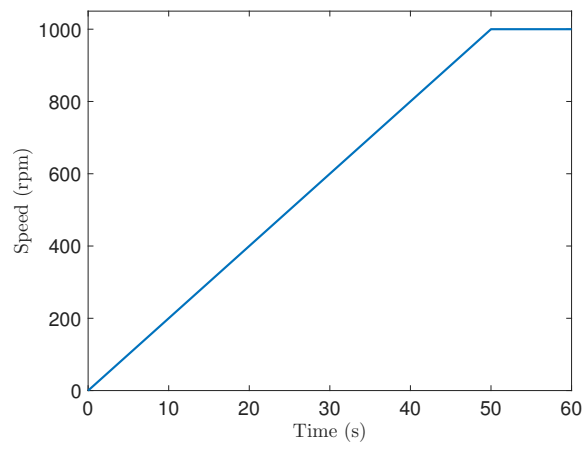

Figure 3: Spin up profile 2.

\section{A. Stationary and rotating frames of reference}

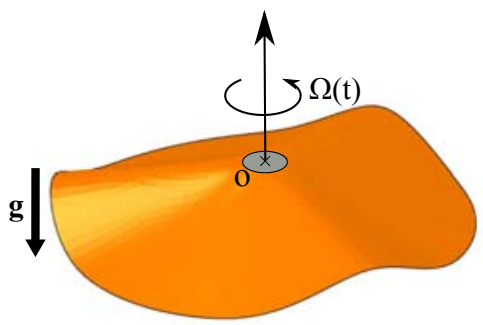

Figure 4: Problem in "Stationary Frame"

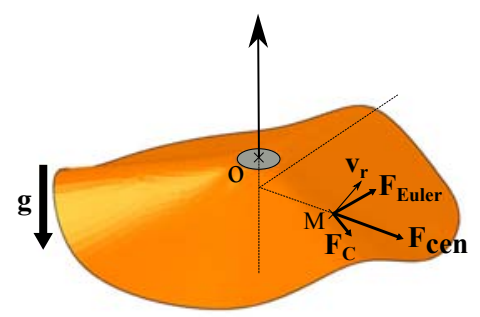

Figure 5: Problem in "Rotating Frame"

We investigated two ways of modeling the spinning behavior using Abaqus/Standard. One can carry out the analysis in the stationary frame, where the hub spins and the whole structure rotates accordingly, or in the rotating frame that follows the hub rotation. A representation of this change of frame is illustrated figures 4 and 5. As in the stationary frame we do not have forces that depend on velocities, the two approaches should give exactly the same results.

In the rotating frame one needs to add the following fictitious forces to gravity:

$$
\begin{aligned}
\mathbf{F}_{\text {cen }} & =-\rho \boldsymbol{\Omega} \times \boldsymbol{\Omega} \times(\mathbf{M}-\mathbf{O}) \\
\mathbf{F}_{\text {Euler }} & =-\rho \frac{d \boldsymbol{\Omega}}{d t} \times(\mathbf{M}-\mathbf{O}) \\
\mathbf{F}_{\mathbf{C}} & =-2 \rho \boldsymbol{\Omega} \times \mathbf{v}_{\mathbf{r}}
\end{aligned}
$$

where:

$\Omega(t)$ is the angular velocity;

$\mathrm{g}$ is the gravity acceleration;

$\mathbf{v}_{r}$ is the velocity in the rotating frame; 
$\rho$ is the density of the membrane;

$\mathbf{F}_{\text {cen }}$ is the centrifugal force;

$\mathbf{F}_{\text {Euler }}$ is the rotary acceleration;

$\mathbf{F}_{\mathbf{C}}$ is the Coriolis force.

These forces are available in the Abaqus library but care is needed in using the built-in library, as different angular velocity-related inputs have to be provided if Abaqus/CAE is used instead of a run file. Hence, we implemented our own centrifugal load and rotary acceleration using the user subroutine DLOAD, and used the Coriolis force implemented in the Abaqus library. To test our own implementation of the centrifugal force, through an Abaqus subroutine, we considered the simplified case of a spinning rigid blade for which we can also derive an analytical solution.

Consider the rigid blade shown in figure 6. It is hinged, with only one degree of freedom allowed, the rotation along an axis perpendicular to a rotating rod. The rod was rotated with an angular velocity linearly increasing from $0 \mathrm{rad} \mathrm{s}^{-1}$ to $12 \mathrm{rad} \mathrm{s}^{-1}$ over a period of $6 \mathrm{~s}$. The dimensions used are in the following table and were chosen to represent the membrane dimensions.

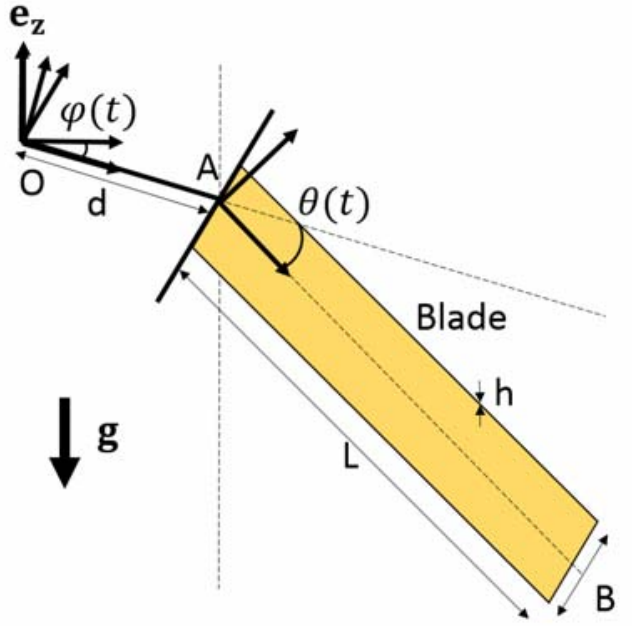

Figure 6: Blade in "Stationary Frame".

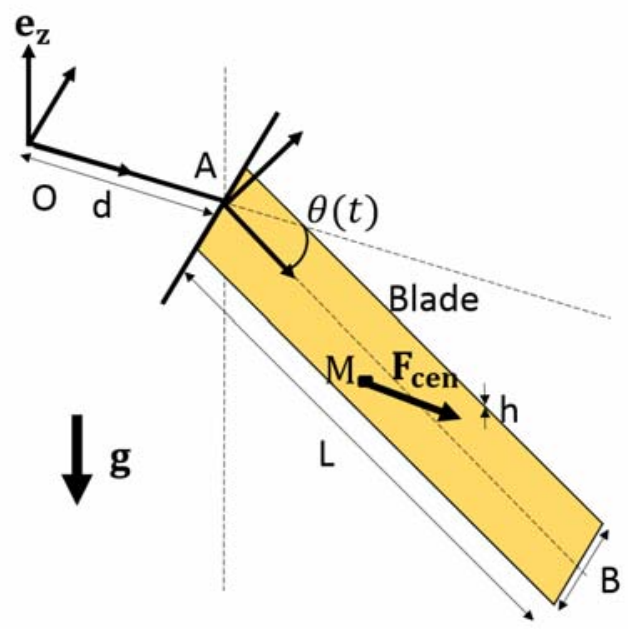

Figure 7: Blade in "Rotating Frame".

\begin{tabular}{|l|c|}
\hline Density $\rho$ & $1420 \mathrm{~kg} \mathrm{~m}^{-3}$ \\
\hline Thickness $h$ & $50 \mu \mathrm{m}$ \\
\hline Length $L$ & $20 \mathrm{~cm}$ \\
\hline Width $B$ & $1 \mathrm{~cm}$ \\
\hline$d$ & $2 \mathrm{~cm}$ \\
\hline$\Omega_{d o t}$ & 2 rad. $s^{-2}$ \\
\hline
\end{tabular}

Table 2: Blade dimensions and properties.

This is a one degree of freedom problem. The unknown is $\theta(t)$ the angle of the blade with the vertical. We simulated this problem in Abaqus in the stationary frame (figure 6) and in the rotating frame (figure 7 ) with Abaqus Centrifugal load and a user subroutine that we had written.

The analytical solution for this case is the solution of the differential equation:

$$
\frac{1}{3} B L^{3} h \rho \ddot{\theta}=-\frac{1}{2} B L^{2} g h \rho \cos \theta-\frac{1}{3} B L^{3} h \Omega_{d o t}^{2} \rho t^{2} \cos \theta \sin \theta=-\frac{B L^{2}}{2} d h \Omega_{d o t}^{2} \rho t^{2} \sin \theta
$$




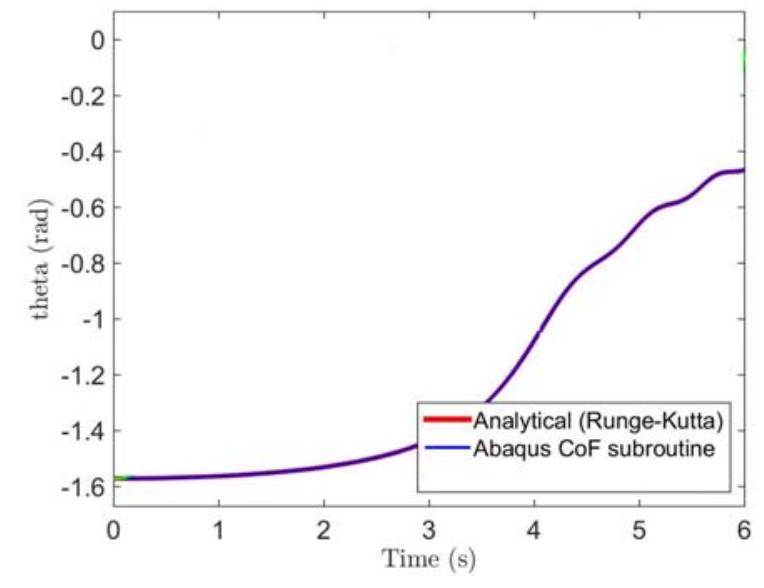

Figure 8: Comparison of analytical solution with Abaqus solution.

The results are shown in figure 8 . The red line is the solution of the differential equation 4 , solved with the Runge-Kutta solver, the blue line is the solution obtained with the subroutine.

There is excellent agreement between our implementation of the centrifugal force and the MATLAB solution for this simple case. Hence from now on we use the implemented subroutine. Note that this case study did not test the rotary acceleration or the Coriolis force as only the centrifugal load affects $\theta$.

Next, we go back to the membrane spin up case and compare the efficiency of the two techniques. We run the simulation in both frames for the full spin up and compared the time to run each simulation in table 3 . The simulations in table 3 were performed on a computer with $2 \operatorname{Intel}(\mathrm{R}) \mathrm{Xeon}(\mathrm{R})$ processors running at $2.33 \mathrm{GHz}$, with 4 cores per processor. For the rest of the simulations we use a computer with 2 processors Intel(R) Core(TM) running at $2.2 \mathrm{GHz}$, with 4 cores.

\begin{tabular}{|l|c|}
\hline Simulation & Total CPU Time $(\mathrm{s})$ \\
\hline Stationary Frame & 302,844 \\
\hline Rotating Frame & 4,335 \\
\hline
\end{tabular}

Table 3: Simulation time in stationary and rotating frames of reference.

In order to compare the two results, we have plotted the strain energy as a global way of comparing the shapes, in figure 9 . Is is also a quantity not affected by the change of frame. Table 3 shows that there is good agreement but the simulation in the rotating frame is two orders of magnitude faster for the same mesh, same integration scheme and time step refinement algorithm. The reason why it is faster is that the time step can be much larger in the rotating frame. For this reason we chose to simulate the spin up in the rotating frame and not in the stationary frame and we will use our implementation of the centrifugal force and rotary acceleration.

\section{B. Equilibrium shape under gravity of stationary membrane}

The first step in the analysis consists in finding the equilibrium shape of the membrane hanging under gravity. We perform this static analysis, considering two cases: with and without the support plate. It is computationally expensive to go through the same shape instabilities that occur during the spin up to find the equilibrium shape. A simpler approach is to apply the gravity preload in two steps. First we impose two boundary conditions in two diametrically opposed points (P1 and P2) as displacements in the $\mathbf{e}_{\mathbf{3}}$ direction of $10 \mathrm{~cm}$ without the plate and until we reach the plate (i.e. $3.4 \mathrm{~cm}$ ) with the plate. Then we relax those constraints and impose the gravity field while maintaining the circumferential position of one point (P1). It is obvious that any rotation of the shape obtained from this process, around $\mathbf{e}_{\mathbf{3}}$, is also an equilibrium shape. 


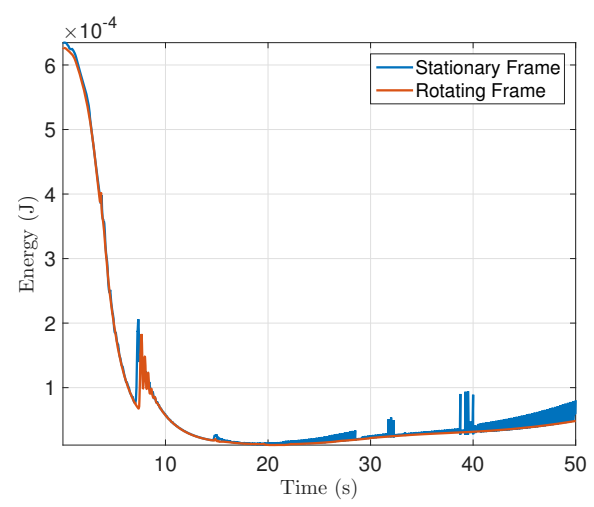

Figure 9: Strain energy in rotating and stationary frames.

This last constraint is to make sure that the solution is unique. This technique converges better than simply imposing a gravity field on an initially flat membrane. The two steps of the preload application are shown in figure 10 and 11, with the support plate, and figures 12 and 13, without the plate. Note that the plate is not shown in these figures.

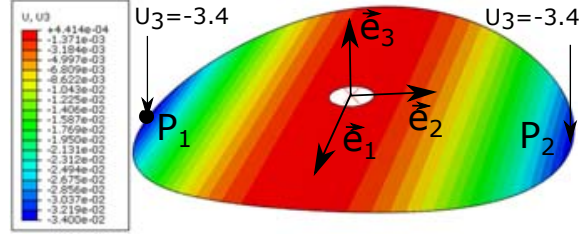

Figure 10: Step 1 with support plate.

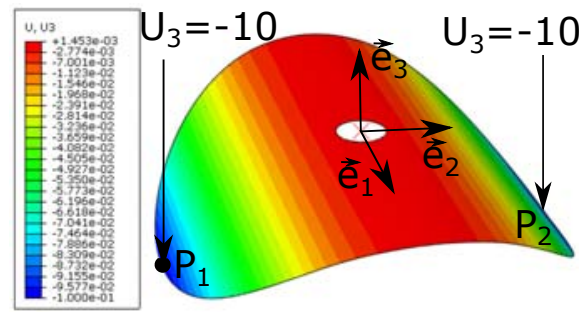

Figure 12: Step 1 without support plate.

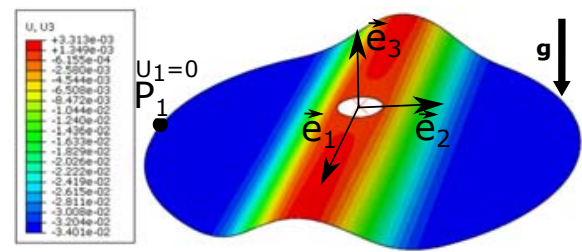

Figure 11: Step 2 with support plate.
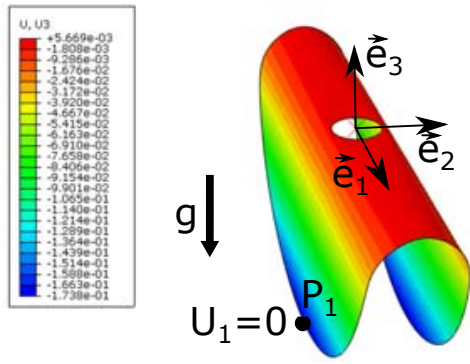

Figure 13: Step 2 without support plate.

\section{Membrane spin up results}

In this section we show the behavior of the membrane during the spin up phase, when it is initially supported by a flat plate. All the results presented were obtained with numerical damping and mesh 4 as described in Section II.E. During spin up the membrane goes through successive, sudden changes of shape. The different numbers of rotationally symmetric waves, $2,4,5,6,7,8,10,11$, can be identified in figure 14 . The final axisymmetric shapes is shown in figure 15 .

Since it is difficult to show the shape of the membrane at each instant, we consider different global parameters related to energy. In particular we look at the strain energy of the membrane, which gives an idea of the global deformation of the structure both in bending and stretching and is shown in figure 16 . During the static analysis in section II.B the membrane mainly bends due to gravity and gains internal energy up to $2.110^{-4} \mathrm{~J}$. When it starts spinning the centrifugal force flattens the structures thus decreasing 


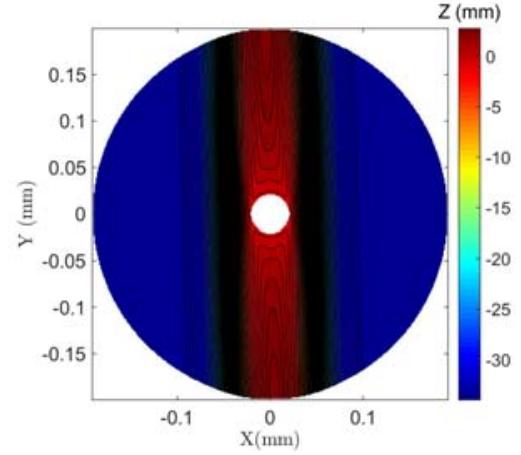

(a) $\Omega=0 \mathrm{rpm}$

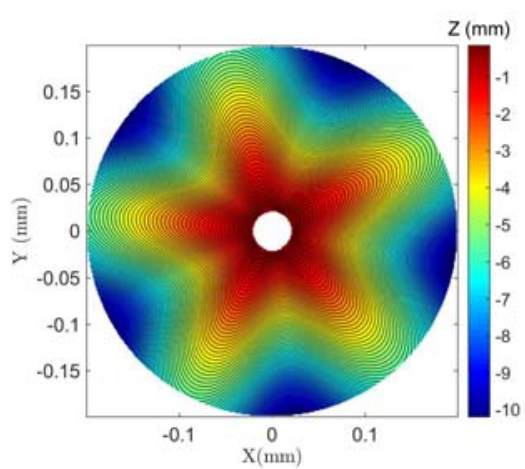

(c) $\Omega=330 \mathrm{rpm}$

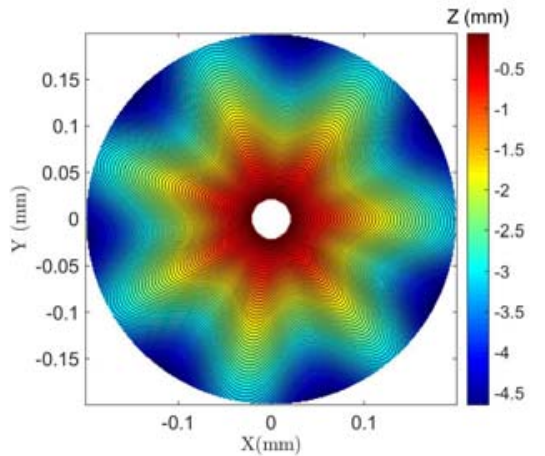

(e) $\Omega=505 \mathrm{rpm}$

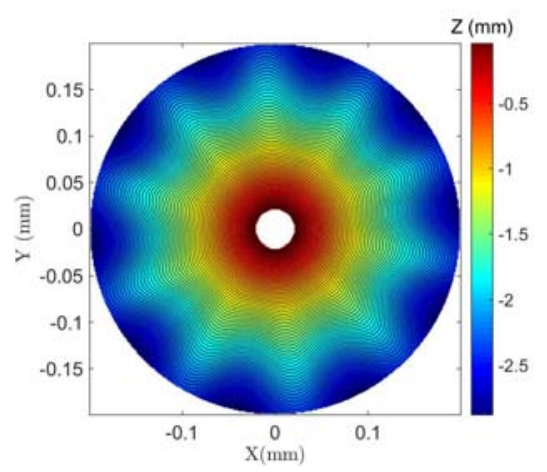

(g) $\Omega=615 \mathrm{rpm}$

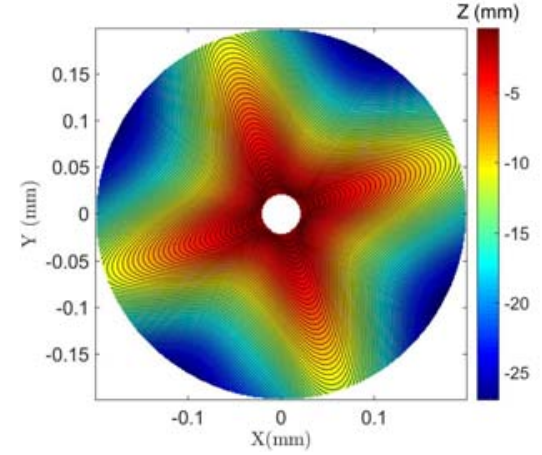

(b) $\Omega=160 \mathrm{rpm}$

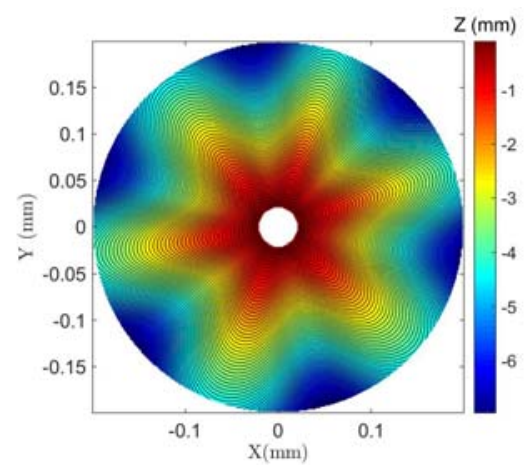

(d) $\Omega=420 \mathrm{rpm}$

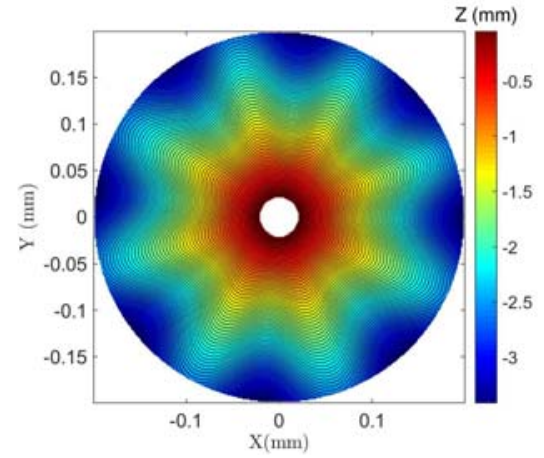

(f) $\Omega=570 \mathrm{rpm}$

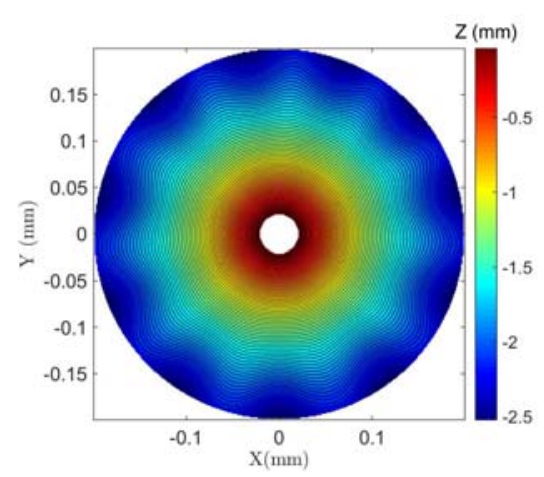

(h) $\Omega=670 \mathrm{rpm}$

Figure 14: Successive shapes of spinning membrane. 


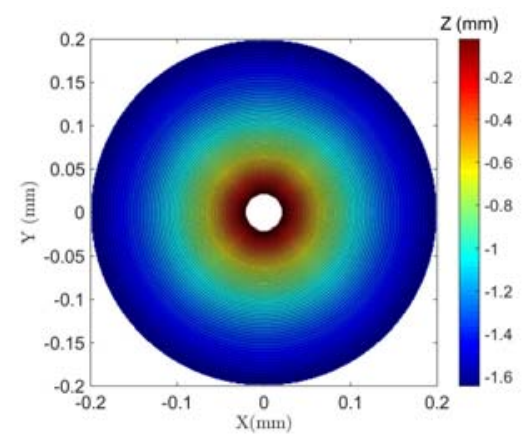

Figure 15: Membrane shape at $\Omega=1000 \mathrm{rpm}$.

the bending energy. The number of rotationally symmetric waves increases. As the bending energy decreases and the membrane is spinning faster the stretching energy becomes dominant in the internal energy which explains the increase of energy after $20 \mathrm{~s}$. The potential energy (strain energy minus external work) is shown in figure 17. Notice the sudden decreases of energy corresponding, to instabilities of the membrane.

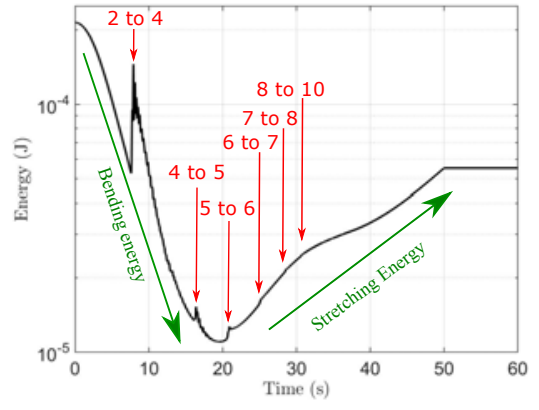

Figure 16: Strain energy.

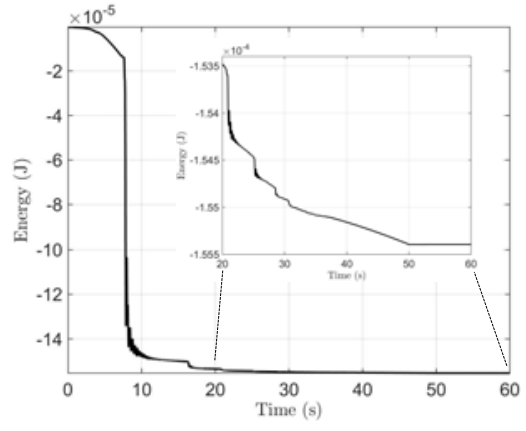

Figure 17: Potential energy.

In the next two parts we will analyze the respective influence of integration scheme, numerical damping, increment tolerance and mesh density. We look into the strain energy, the kinetic energy, the external work and the total energy. We also compare the time for each simulation. We will see that without any source of damping it is difficult to achieve energy convergence. We will finally add stiffness proportional material damping.

\section{Numerical damping and time increment}

We used the two main implicit integration schemes available in Abaqus/Standard: ${ }^{13}$ Euler-Backward (EB) and Hilber-Hughes-Taylor (HHT) which is a modified Newmark method that averages the internal and external forces between adjacent time steps. The first is mainly used for quasi-static simulations. The second can be used for a wide range of applications, with different amount of numerical damping depending on the coefficient $\alpha(-0.5 \leq \alpha \leq 0)$. Smaller values of $\alpha$ correspond to more accurate simulations, which take longer to run; larger values of $\alpha$ lead to faster simulations but less accurate (more dissipative).

We also used Abaqus time step refinement algorithms. For transient fidelity simulations the algorithm is based on the half-increment residual and is constrained by the half increment tolerance. Smaller tolerance means finer time steps and longer, more accurate simulations. The half increment tolerance and $\alpha$ are the parameters that we changed in the simulations. We also used a rather coarse mesh, corresponding to Mesh1 in Section E.

We compared the results in terms of energy in the plot in figure 18 and the time of each simulation in table 4 for the five following cases (some of which are default Abaqus/Standard parameters):

- Case 1: EB (default Quasi-Static option in Abaqus/Standard)

- Case 2: HHT- $\alpha=-0.41 t_{\max }=$ Time step/10 (defaults Moderate Dissipation option in Abaqus/Standard) 
- Case 3: HHT- $\alpha=-0.05 t_{\max }=$ Time step/100 (default Transient Fidelity option in Abaqus/Standard). The time step refinement algorithm is base on the half increment residual with a scaling factor of 1000 .

- Case 4: HHT- $\alpha=-0.01$ HAFTOL=0.1 (absolute value of the tolerance for the half increment residual algorithm)

- Case 5: HHT- $\alpha=-0.001$ HAFTOL=0.1 (absolute value of the tolerance for the half increment residual algorithm)

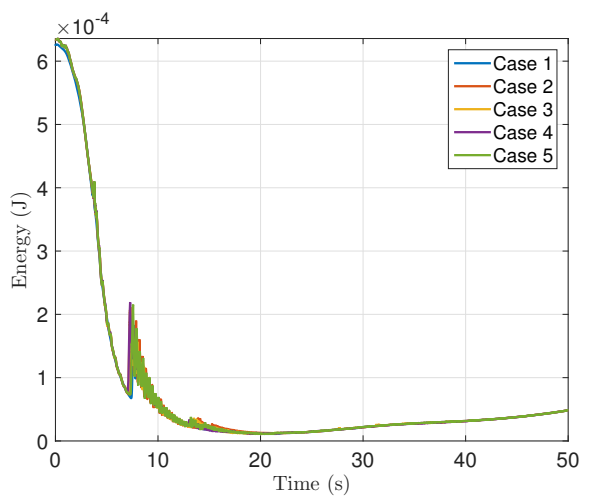

(a) Strain Energy.

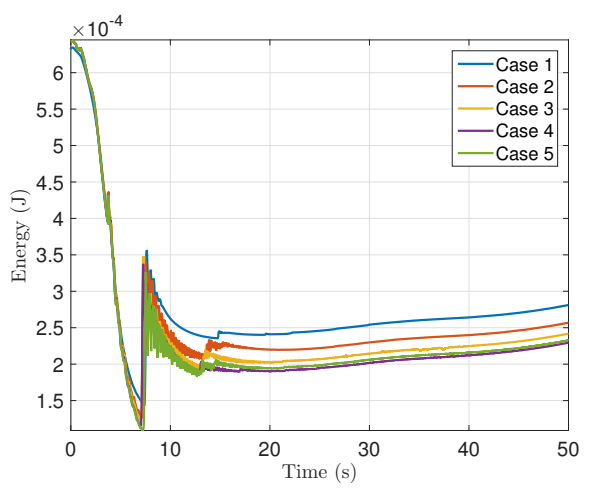

(c) External Work.

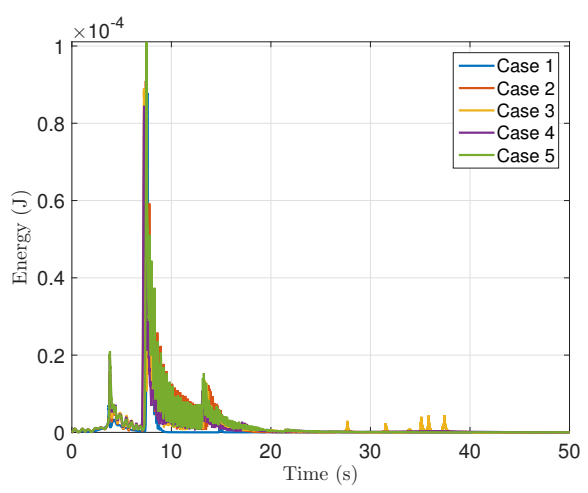

(b) Kinetic Energy.

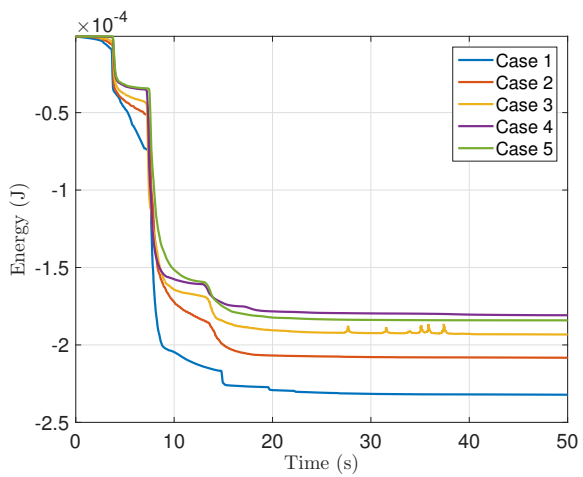

(d) Total Energy.

Figure 18: Plots of energy components.

We noticed improved energy convergence, but also longer run times. For case 5 (the most energy conservative case) the energy loss is $20 \%$ better than in case 1 (the least conservative one) while the run time is 63 time longer.

\begin{tabular}{|l|c|}
\hline Simulation & Time $(\mathrm{s})$ \\
\hline Case 1 & 4,335 \\
\hline Case 2 & 16,140 \\
\hline Case 3 & 48,982 \\
\hline Case 4 & 107,484 \\
\hline Case 5 & 263,229 \\
\hline
\end{tabular}

Table 4: Simulation times. 


\section{E. Mesh effects}

We used the parameters of case 3 , which provided the best combination between energy conservation and run time, to examine the effects of changing the mesh density. We considered different meshes, increasing the mesh density around the hub by varying the number of elements along each of the edges 1,2 and 3 , as shown in figures 19 and 20 :

- Mesh 1: Seed 20-100, total number nodes: 1746

- Mesh 2: Seed 40-200, total number of nodes: 5098

- Mesh 3: Seed 80-60-200, total number nodes: 5735

- Mesh 4: seed 150-100-200, total number nodes: 11895

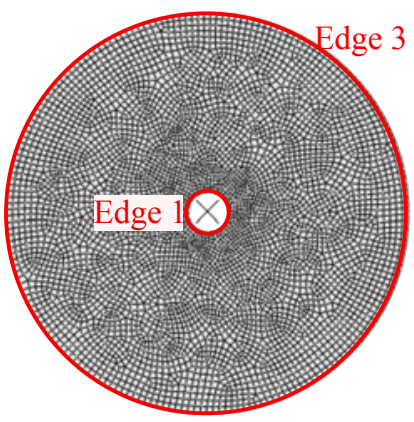

Figure 19: Mesh 2 and edges 1 and 3.

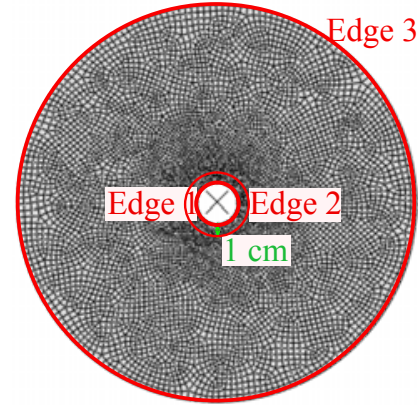

Figure 20: Mesh 4 and edges 1, 2 and 3.

The density of the mesh influences significantly the results. Finer meshes lead to more chaotic simulations and more gradual transitions between successive shapes of the membrane. We illustrate this by comparing the strain energy in figure 21. Note that the energy oscillates more and more. We compare the run time of each simulation in table 5 .

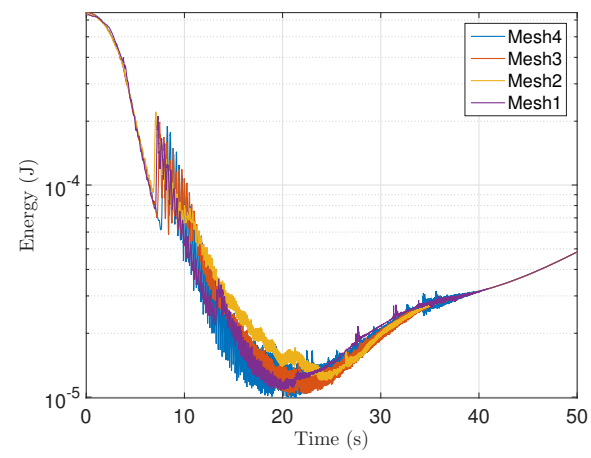

Figure 21: Strain energy for different meshes.

\begin{tabular}{|l|c|}
\hline Mesh & time (s) \\
\hline Mesh 1 & 48,982 \\
\hline Mesh 2 & 278,003 \\
\hline Mesh 3 & 274,110 \\
\hline Mesh 4 & 492,219 \\
\hline
\end{tabular}

Table 5: Simulation time for each mesh. 
These studies have shown that it is difficult to capture all the wavelengths and frequency of the dynamics across the instabilities of the structure. Not only energy convergence is difficult to achieve, but also convergence according to the mesh density. The successive shapes are different depending on the mesh and the integration scheme and the time associated with each snap changes.

\section{F. Effects of material damping}

Next, we tried to obtain more stable results by including material damping while using a less conservative integration scheme. We also introduced the support plate in the simulation, and a flat angular velocity plateau at the end of the simulation, for a closer match to the conditions of the experiments.

We introduced stiffness proportional damping of $0.0008 \mathrm{~s}^{-1}$. Frictionless contact between the membrane and the support plate is introduced. We used the moderate dissipation parameters corresponding to case 2 in section II.D. The transient fidelity option doesn't work properly when there is contact. Also, having introduced material damping high frequency dynamics were suppressed. We considered three different meshes. Meshes 3 and 4 introduced before and mesh 5 to check the convergence. Mesh 5 has seeds 200-100-200 and 12,347 nodes in total.

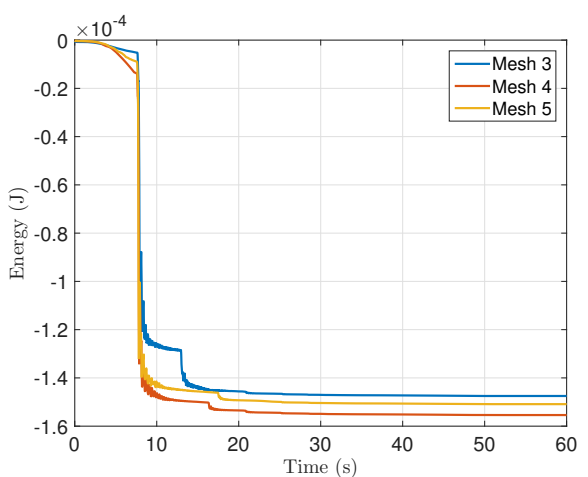

(a) Potential Energy

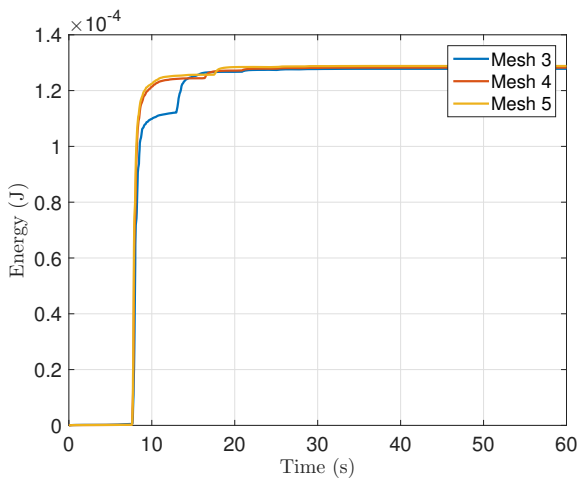

(c) Viscous Work

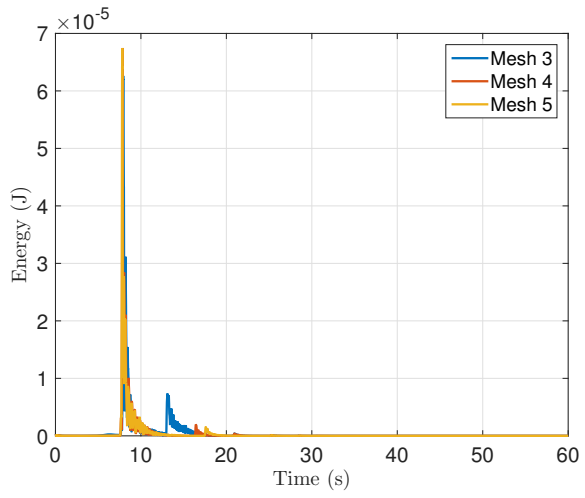

(b) Kinetic Energy

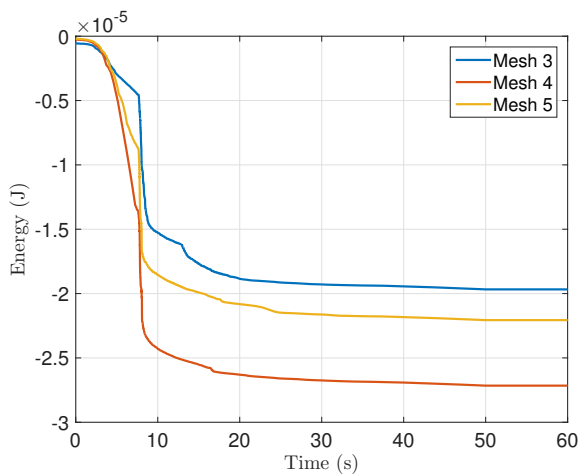

(d) Total Energy

Figure 22: Energy components.

There are clear differences between the results obtained with mesh 3 and 4 . As a matter of fact the first snap is from 2 to 3 waves for the case of mesh 3, while for meshes 4 and 5 it is from 2 to 4 waves. The results with mesh 4 and 5 are quite similar, even though a closer look to the potential energy shows a difference of about $3 \%$ on the final potential energy, for example (figure $22 a$ ).

For the comparison between experiments and simulations in section IV we use the results obtained with mesh 4 . The results are presented figure 22 and the run times in table 6 . We notice that the total energy (figure $22 d$ ) loss is now less than $3.10^{-5}$ which is an order of magnitude less than in the case without damping. The energy is dissipated by material damping, as seen in figure $22 \mathrm{c}$. It is difficult to compare the run times as in this set of simulations we have included frictionless contact with the support plate. It is surprising that 
it is more than twice as expensive to run the simulation with mesh 5 than mesh 4 even if the increase in the number nodes is only $4 \%$.

\begin{tabular}{|l|c|}
\hline Mesh & time (s) \\
\hline Mesh 3 & 512,994 \\
\hline Mesh 4 & 603,908 \\
\hline Mesh 5 & 1599,120 \\
\hline
\end{tabular}

Table 6: Simulation time for each mesh.

\section{Experiments}

We studied membrane spin up experimentally, to assess the accuracy of the simulations and to develop experimental measurement techniques using stereo DIC.

\section{A. Setup description}

We have developed a setup that enables spinning the membrane in vacuum. It is also possible to shake the membrane but shaking was not applied in the experiments described in this paper.

The main challenges of setting up a lab experiment was to decouple the source of rotation (electric motor) from the vertical motion (shaker). We decoupled rotation and linear motion with a system of gears and ball splines as shown in figure 23. A reflective support plate was used to provide uniform lighting during image acquisition for DIC. The whole setup is under vacuum, as shown in figure 24 and reaches a vacuum level of $6.5 \mathrm{kPa}$. We can see the stereo DIC system on top, the vacuum box with the membrane setup inside, in the middle and the vacuum pump at the bottom. There also are 4 halogen lights around the setup. We use stereo Digital Image Correlation (DIC) to record the time variation of the shape and displacements of the membrane.

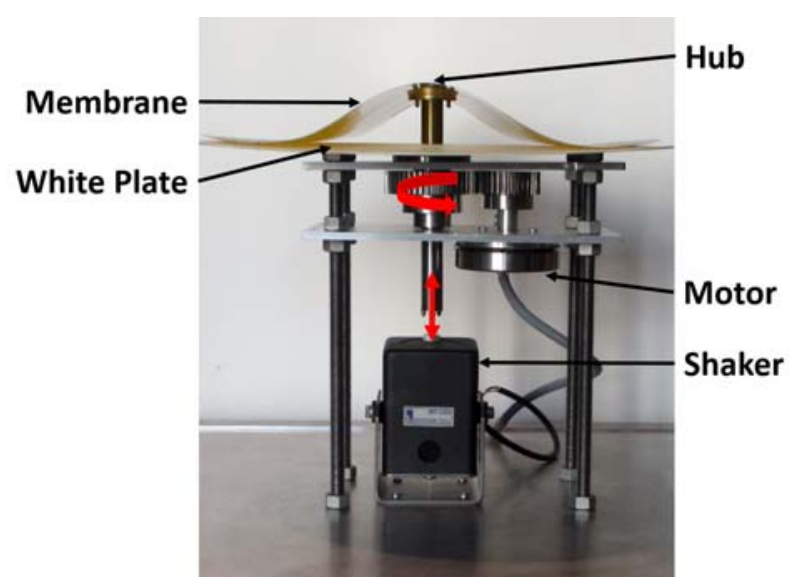

Figure 23: Mechanical setup.

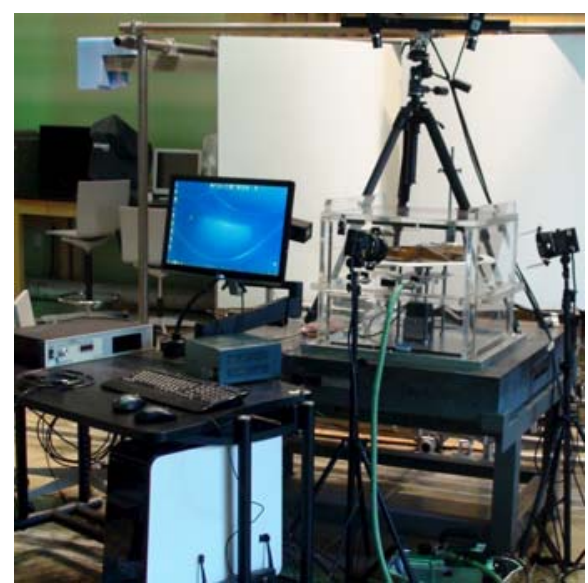

Figure 24: Complete test setup.

\section{B. Measurement technique}

Our stereo DIC system consists of two Point Grey Grasshopper cameras GRAS-50S5M-C with a resolution of $2448 \times 2048,3.45 \mu \mathrm{m}$ pixel size and a gain range between $0 \mathrm{~dB}$ and $24 \mathrm{~dB}$. We used lenses with a focal length of $12 \mathrm{~mm}$ to image our sample. The stereo angle was about $25^{\circ}$. We used the commercial software Vic3D 2010 by Correlated Solutions to correlate the images. The membrane is $40 \mathrm{~cm}$ in diameter and the field of view is about $50 \mathrm{~cm}$ which corresponds to a pixel size of $200 \mu \mathrm{m}$. We hand drew speckles about $3 \mathrm{~mm}$ 
wide (15 px) on the membrane, with a black marker. We illuminated the sample from below by reflecting 4 halogen lights on a white plate as shown in figure 25.

The membrane spins at an angular velocity of up to $1000 \mathrm{rpm}$. To reduce the blur on the outer edge, we used a short exposure time. The DIC system is able to achieve sub pixel resolution and the exposure time should be chosen such as to keep motion below $0.01 \mathrm{px}$. Even with 4 halogen lights we could not go as low as this and instead took images at $63 \mu \mathrm{s}$, which corresponds to a motion of $1 \mathrm{~mm}$ ( $5 \mathrm{px})$ on the very edge and thus creates blur. A better lighting system would have been required for faster exposures .

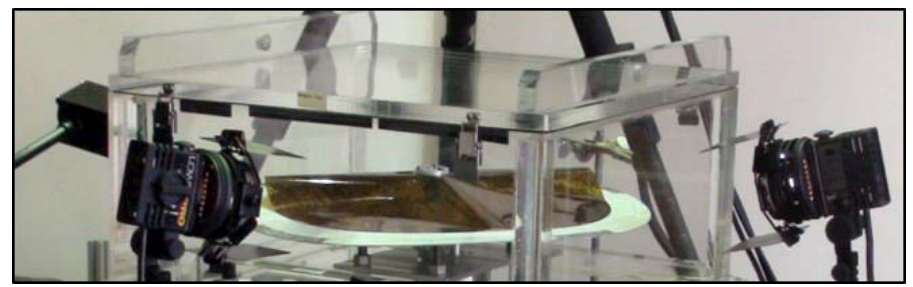

Figure 25: Lighting arrangement.

Kapton has an orange color and is transparent, which makes it difficult to obtain images with high contrast. Black Kapton with white speckles is an alternative option for future experiments.

One of the challenges when studying very flexible structures is to get an unstressed reference image. Under gravity this state is difficult to find. We assumed that the unstressed membrane is flat and we supported the membrane on a flat aluminum plate, to take our reference image.

Another challenge is to deal with the optical distortions created by the thick $(2,54 \mathrm{~cm})$ acrylic lid of the vacuum box. Each camera is looking through the lid at a different angle and at a different location. Each camera is thus going to see a different rigid body motion and a different optical distortion of the membrane due to the lid. We corrected for the different rigid body motions by correcting the calibration of the DIC system. The steps for the stereo DIC processing are as follows:

1. Calibration with membrane placed below the acrylic lid.

2. Take images of the membrane from two points at a known distance.

3. Using these two points optimize the camera angles for the lowest projection error to form an epipolar line due to the refractive influence of the lid.

4. Run the correlation.

This technique does not correct the optical distortion but reduces the projection error due to the rigid body motion part that the optical distortion introduced on each camera.

We performed a "stationary images" test to estimate the errors due to lighting, speckles and contrast. To this end we took two successive images of the same stationary sample and run a correlation. Any displacement field between these two images correspond to an error, of course this error does not include the blurring due to rotation of the membrane. We obtained a random error with zero mean and a maximum amplitude of about $20 \mu \mathrm{m}$ on the out-of-plane displacement component.

The parameters used for the correlation were subset of 45 pixels (corresponding to a square with side length of about $1 \mathrm{~cm}$ ) and density of 3 pixels (meaning that we computed a data point every 3 pixels). Such a high density of points is not needed, but it seemed to help the software correlate the images, despite the large amplitude rotation between the reference image and the current image. We used the following parameters in the software: the shape functions assume linear deformation within subset with Gaussian weights, the pixel interpolation uses 8-tap splines, the minimization algorithm used a zero-normalized square difference (unaffected by offset plus scale change is lighting). Each image had an average projection error of about 0.06 pixel. Choosing a large subset helped the correlation and decreased the noise but also decreased the spatial resolution by averaging the deformation linearly within a subset during matching. Once again this choice was driven by the difficulty of correlating large deformations and the low contrast in the images (meaning high noise).

Altogether, with the current setup we estimate a resolution of about $1 \mathrm{~cm}$ and a precision of $\pm 20 \mu \mathrm{m}$. The bias was not estimated. 


\section{Experimental results}

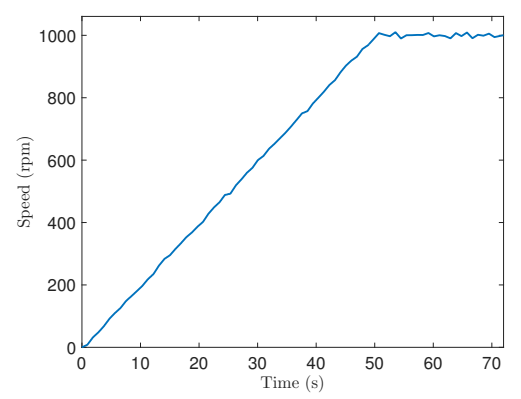

Figure 26: Angular velocity profile.

The experimental spin up curve is shown in figure 26. Images obtained at different times, and hence associated with different velocities, are shown in figure 27. Note that these images are unsmoothed, and without any extrapolation near the edges (to get data up to the edge of the sample). These images were despun using speckles on the rigid hub and by calculating for each frame the average rigid body motions on the hub. All the images in figure 27 are thus in the rotating frame.

Notice the close resemblance of these images to the predictions from the simulations. The final shapes though, shown in figure 29, are not fully axisymmetric as predicted. This could be due to the imperfections of the setup, e.g. vibrations of the shaft, the aerodynamic excitation due to the remaining air in the box, the slight initial curvature of the membrane, or the projection error due to optical distortions associated with the acrylic lid.

\section{Comparison of Experiments and Simulations}

We ran two experiments and compared the results to the damped simulation with high density mesh (mesh 4) and low numerical damping. Here we compare three quantities: the number of rotationally symmetric waves according to angular velocity, the average displacement over the membrane and the bending strain energy. The bias introduced by the acrylic lid, the non flat reference image and the different post processing techniques is not considered.

It is important to note that the experimentally measured deflections and bending energies have not been smoothed, whereas the shapes and curvatures are. A problem when evaluating global quantities over the whole membrane is that the DIC does not correlate up to the edges. We used the MATLAB function "scatteredInterpolant" with linear extrapolation to evaluate the scatter data on a uniform grid up to the edges of the membrane. Finally we simply integrated by multiplying by a Boolean operator and added the matrix components, each multiplied by the size of each element.

\section{A. Number of waves and average displacement}

We determined the angular velocities at which the instabilities occur up to 10 waves formed; a higher number of waves was difficult to estimate in the experimentally obtained images. We defined the average magnitude of displacement (not just the vertical component of displacement) to avoid projection errors, relative to the initial shape:

$$
A v=\frac{1}{\pi\left(b^{2}-a^{2}\right)} \iint_{\Omega_{f}} \sqrt{U\left(x_{f}, y_{f}\right)^{2}+V\left(x_{f}, y_{f}\right)^{2}+W\left(x_{f}, y_{f}\right)^{2}} d x_{f} d y_{f}
$$

where: $\left(x_{f}, y_{f}\right)$ are the coordinates in the reference configuration; $(U, V, W)$ are the components of displacement; and $\Omega_{f}$ is the membrane domain in the reference state.

Before evaluating the average magnitude of displacement we smoothed the images using a decay filter which is a $90 \%$ center-weighted Gaussian filter with size of 31 . This means that we averaged the displacements over a square of $93 \mathrm{px} * 93 \mathrm{px}$.

The number of waves observed in the experiments and average magnitude of displacements (without rigid body motion) obtained experimentally and numerically are figures 30 and 31. 


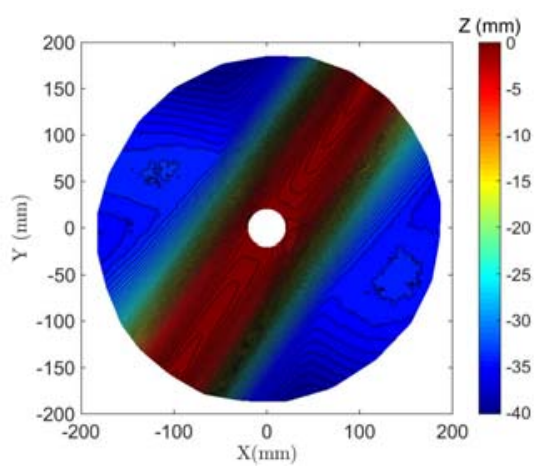

(a) $\Omega=0 \mathrm{rpm}$

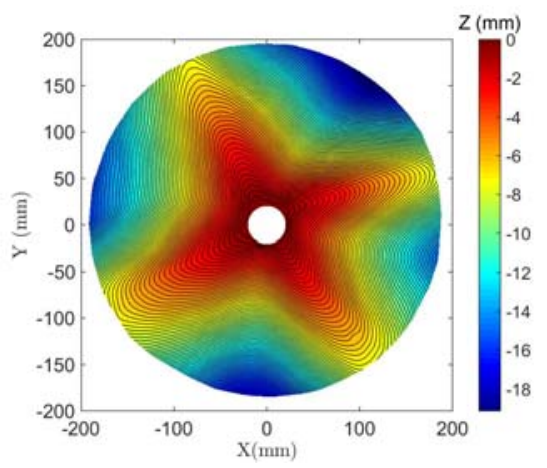

(c) $\Omega=260 \mathrm{rpm}$

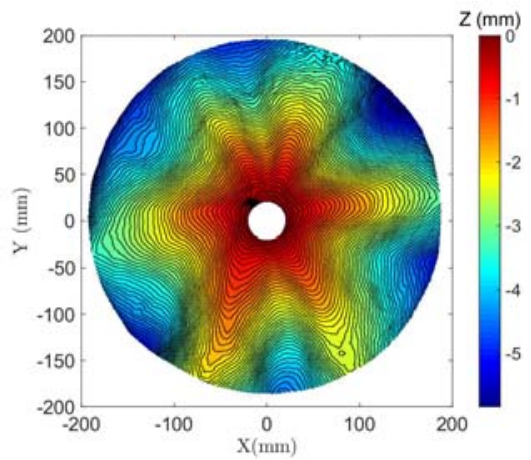

(e) $\Omega=515 \mathrm{rpm}$

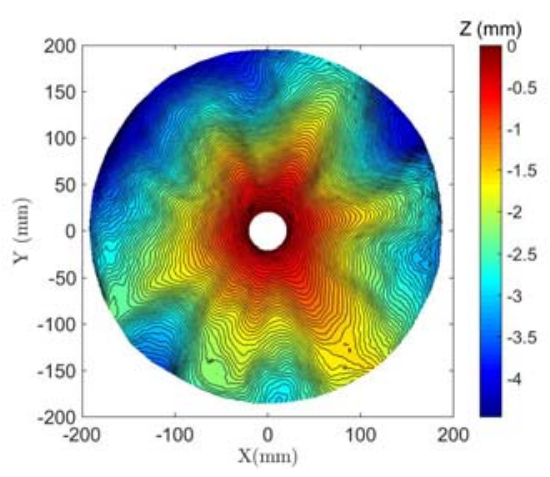

(g) $\Omega=595 \mathrm{rpm}$

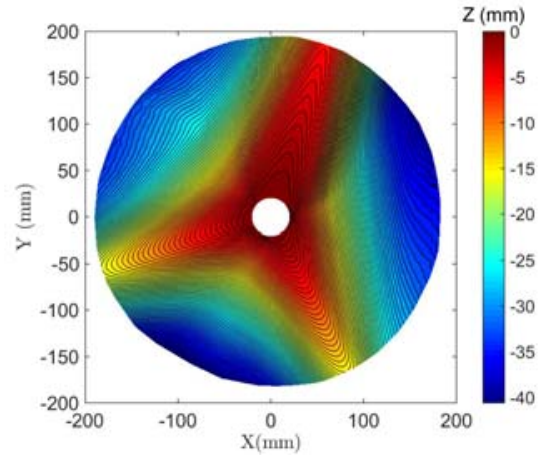

(b) $\Omega=120 \mathrm{rpm}$

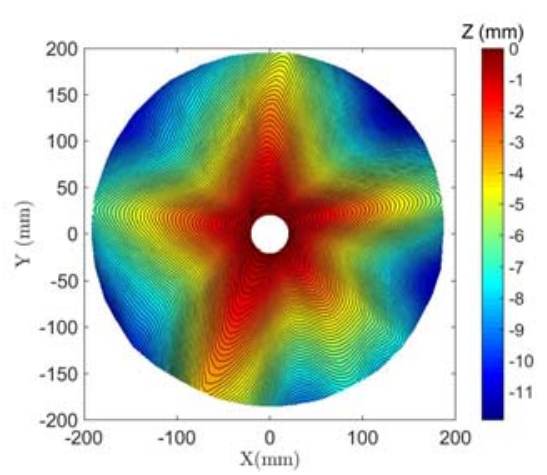

(d) $\Omega=475 \mathrm{rpm}$

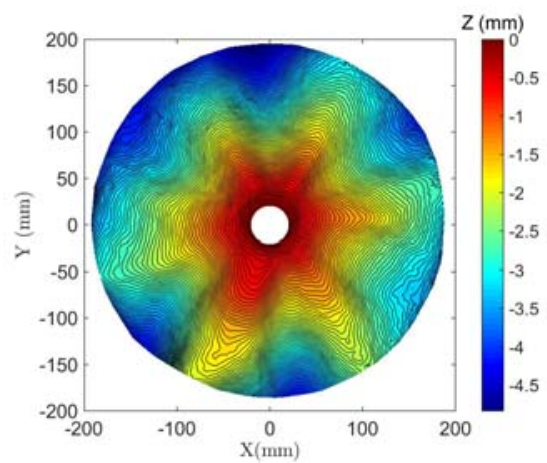

(f) $\Omega=575 \mathrm{rpm}$

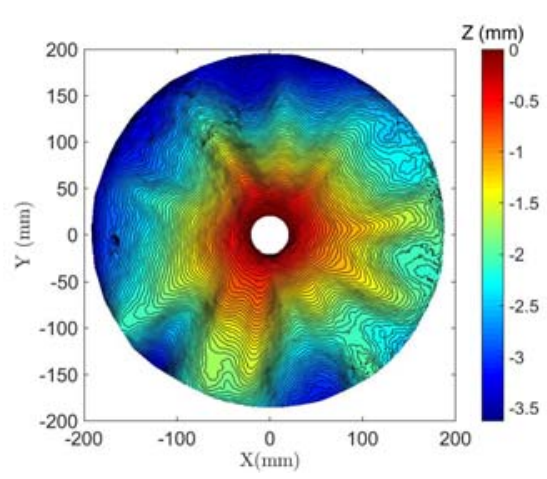

(h) $\Omega=600 \mathrm{rpm}$

Figure 27: Successive shapes during spin-up. 


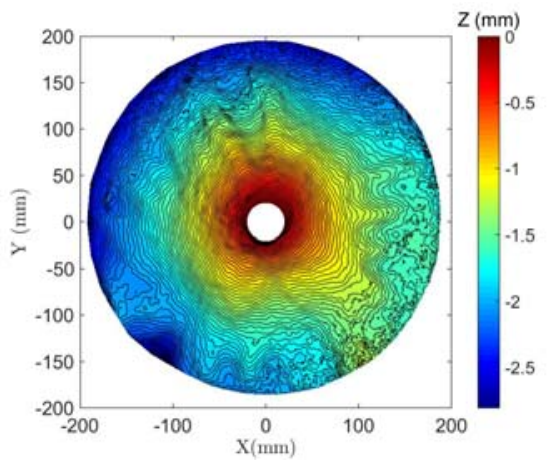

(a) $\Omega=860 \mathrm{rpm}$

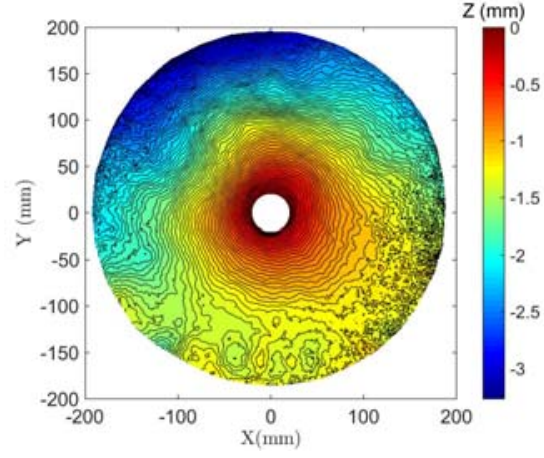

(b) $\Omega=1000 \mathrm{rpm}$

Figure 29: Final shapes at the end of spin-up.

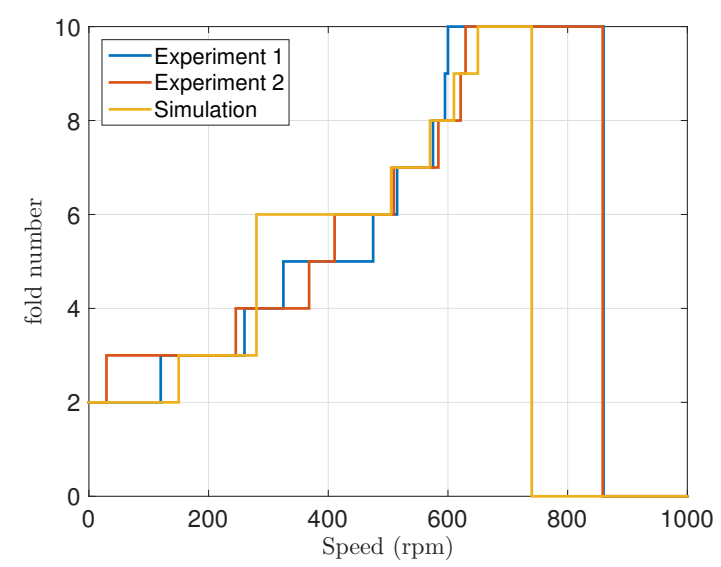

Figure 30: Number of waves.

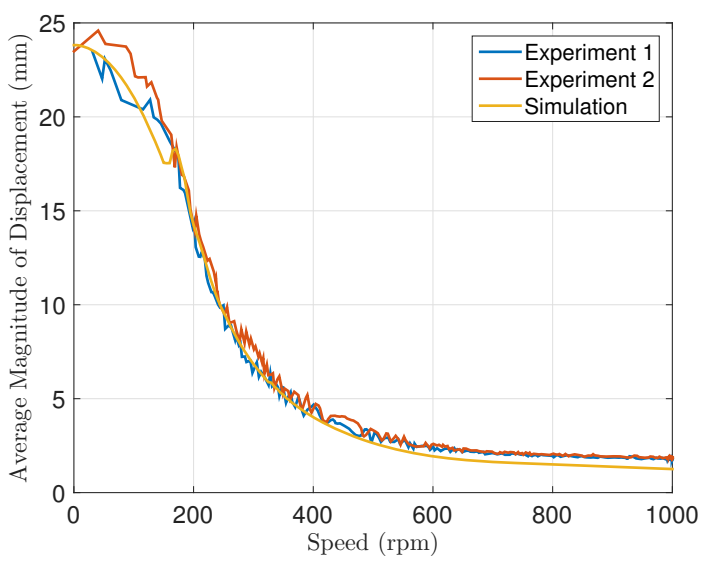

Figure 31: Average deflection.

Figures 30 and 31 show a good agreement between simulations and experiments. The discrepancy at the beginning of the spin up is likely due to errors in the position of the support plate or friction between the plate and the membrane. More general errors can come from imperfections in the membrane, measurement noise, resolution and bias, remaining air in the box, or material damping estimation in the simulations. 


\section{B. Bending energy}

The elastic strain energy $U_{P}$ of a Kirchhoff plate is given by:

$$
\begin{aligned}
U_{P}= & -\frac{1}{2} \frac{E h}{1-\nu^{2}} \iint_{\Omega_{f}}\left(\epsilon_{x, 0}^{2}+\epsilon_{y, 0}^{2}+2 \nu \epsilon_{x, 0} \epsilon_{y, 0}+\frac{1-\nu}{2} \gamma_{x y, 0}^{2}\right) d x_{f} d y_{f} \\
& +\frac{1}{2} \frac{E h^{3}}{12\left(1-\nu^{2}\right)} \iint_{\Omega_{f}}\left(\kappa_{x}^{2}+\kappa_{y}^{2}+2 \nu \kappa_{x} \kappa_{y}+\frac{1-\nu}{2} \kappa_{x y}^{2}\right) d x_{f} d y_{f}
\end{aligned}
$$

where:

$\left(\epsilon_{x, 0}, \epsilon_{y, 0}, \gamma_{x y, 0}\right)$ are the mid-plane strain components;

$\left(\kappa_{x}, \kappa_{y}, \kappa_{x y}\right)$ are the curvatures.

The first term of equation 6 is the membrane or stretching energy and the second term is the bending energy. Our current experiments are not sufficiently precise to evaluate the strains accurately. Hence, we only consider the bending part of the strain energy which is dominant at the beginning of the spin up. The curvatures are evaluated from the position vectors using a decay filter and a filter size of 51 (to smooth the noise). This means that we average the curvature over a square of $153 \mathrm{px} \times 153 \mathrm{px}$. Our spatial resolution decreases significantly but so does the noise level.

The results are presented in figure 32. The experiments give different results for $\Omega \leq 200 \mathrm{rpm}$ but show very similar trends for the rest of the spin up. However, the final value of the bending energy is an order of magnitude higher in the experiment than in the simulation. This could be due to the noise in the experiment as in the bending energy evaluation we sum the square of the noise on the whole membrane. Unfortunately the noise in the curve is too big and the temporal resolution too small to see any jumps in bending energy.

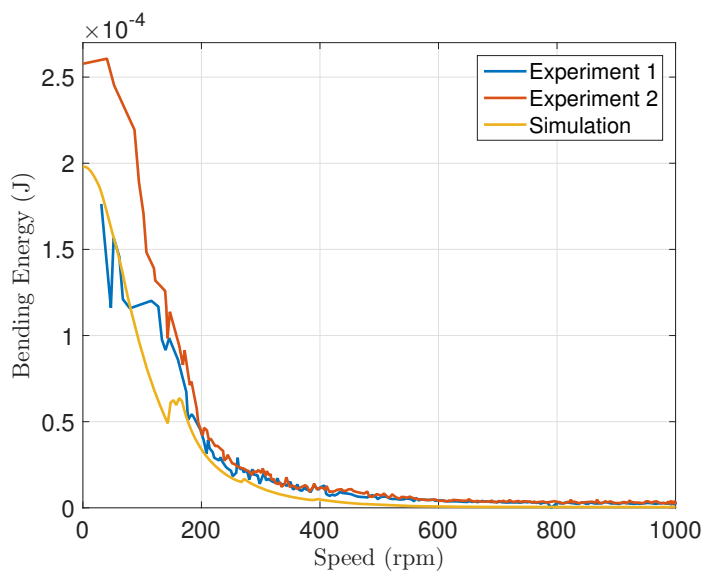

Figure 32: Bending strain energy.

\section{Conclusion}

It has been shown that when a membrane is spun up in a normal gravity field, it goes through successive sudden changes of shapes characteristic of shape instabilities. The average deflection of the membrane decreases during the spin up while the number of rotational symmetric waves increases until the membrane becomes axisymmetric.

We have studied this problem both experimentally and numerically and found quite good agreement between the two approaches. The simulations are fully-integrated, nonlinear dynamic implicit simulations. We have presented techniques to simulate accurately the instabilities of the membrane. We have found that to stabilize the simulations it is beneficial to introduce a dissipation mechanism and to include stiffness proportional material damping. We have developed an experimental setup and measurement techniques using DIC to capture the deformation of the membrane during the spin up. It is challenging to take high quality pictures of a transparent membrane spinning at $1000 \mathrm{rpm}$. We developed a lighting method to 
mitigate these challenges and obtained good measurements of the deformed shape of the membrane. We were able to compare experiments and simulations by means of three quantities: number of waves, average deflection and bending energy.

\section{Acknowledgments}

We thank Professor Sigrid Leyendecker and Tristan Schlogl for helpful discussions and advice. Financial support from the Northrop Grumman Corporation is gratefully acknowledged.

\section{References}

${ }^{1}$ H. Sawada, O. Mori; N. Okuizumi, Y. Shirasawa, Y. Miyazaki, M. Natori, S. Matunaga, H. Furuya, H. Sakamoto. Mission Report on The Solar Power Sail Deployment Demonstration of IKAROS. 52nd AIAA/ASME/ASCE/AHS/ASC Structures, Structural Dynamics and Materials Conference 2011.

${ }^{2}$ R.C. Benson, D.B. Bogy. Deflection of a Very Flexible Spinning Disk Due to a Stationary Transverse Load . Journal of Applied mechanics, Vol. 45, September 1978.

${ }^{3}$ M. Delapierre, S. Pellegrino Spin-Stabilized Membrane Antenna Structures 2nd AIAA Spacecraft Structures Conference, 5-8 January 2015, Kissimmee, FL.

${ }^{4}$ W. Eversman, Transverse Vibrations of a Clamped Spinning Membrane. AIAA Journal, Vol. 6, No. 7, 1968, pp. 1395-1397.

${ }^{5}$ J.G. Simmonds, The transverse vibration of a flat spinning membrane. Journal of the Aerospace Sciences, Vol. 29 , No. 1, 1962, pp. 16-18.

${ }^{6}$ N. Okuizumi, Forced vibration experiments of a rotating extremely thin circular membrane, Mechanical Engineering Journal, Vol.1, No. 5, 2014.

${ }^{7}$ N. Okuizumi, Equilibrium of a Rotating Circular Membrane under Transverse Distributed Load, Journal of System Design and Dynamics, Vol.1, No.1, 2007.

${ }^{8}$ N. Okuizumi, Vibration Mode Analysis of a Rotating Circular Membrane under Transverse Distributed Load, Journal of System Design and Dynamics, Vol.3, No.1, 2009.

${ }^{9}$ N. Triantafyllidis, Stability of solids: From structures to materials. Lecture Notes, Ecole Polytechnique, 2011.

${ }^{10}$ C. Kane, J. E. Marsden, M. Ortiz and M. West, Variational integrators and the Newmark algorithm for conservative and dissipative mechanical systems. International Journal for numerical methods in engineering 2000;49:1295-1325.

${ }^{11}$ S. Kukathasan, Vibration of Space Membrane Structures. Cambridge University PhD, 2003.

${ }^{12}$ C. Liu, M.L. Lovato, K.D. Clarke, D.J. Alexander, and W.R. Blumenthal Miniature Bulge test and energy release rate in HIPed aluminum/aluminum interfacial fracture. Materials Science and Technology Division, Los Alamos National Laboratory. Los Alamos, NM 87545, USA.

${ }^{13}$ Abaqus Theory Manual.

${ }^{14}$ M. Amabili, Nonlinear Vibrations and Stability of Shells and Plates. Cambridge University Press, 2008.

${ }^{15}$ M.A. Sutton, Jean-Jose Orteau, H. W. Schreier Image Correlation for Shape, Motion and Deformation Measurements. Springer, 2009.

${ }^{16}$ P.L. Reu Introduction to Digital Image Correlation: Best practices and Applications. Society for Experimental Mechanics, 2015.

${ }^{17}$ P.L. Reu A Realistic Error Budget for Two Dimension Digital Image Correlation. Sandia National Laboratories, PO Box 5800, Albuquerque, NM 87185. 